\title{
ON A GENERALIZATION OF ALTERNATIVE AND LIE RINGS
}

\author{
BY \\ ERWIN KLEINFELD( $\left.{ }^{1}\right)$
}

\begin{abstract}
Alternative as well as Lie rings satisfy all of the following four identities: (i) $\left(x^{2}, y, z\right)=x(x, y, z)+(x, y, z) x$, (ii) $\left(x, y^{2}, z\right)=y(x, y, z)+(x, y, z) y$, (iii) $\left(x, y, z^{2}\right)$ $=z(x, y, z)+(x, y, z) z$, (iv) $(x, x, x)=0$, where the associator $(a, b, c)$ is defined by $(a, b, c)=(a b) c-a(b c)$. If $R$ is a ring of characteristic different from two and satisfies (iv) and any two of the first three identities, then it is shown that a necessary and sufficient condition for $R$ to be alternative is that whenever $a, b, c$ are contained in a subring $S$ of $R$ which can be generated by two elements and whenever $(a, b, c)^{2}=0$, then $(a, b, c)=0$.
\end{abstract}

Introduction. Alternative rings are known to satisfy the following four identities:

(i) $\left(x^{2}, y, z\right)=x \circ(x, y, z)$,

(ii) $\left(x, y^{2}, z\right)=y \circ(x, y, z)$,

(iii) $\left(x, y, z^{2}\right)=z \circ(x, y, z)$,

(iv) $(x, x, x)=0$,

where the associator $(a, b, c)$ is defined by $(a, b, c)=(a b) c-a(b c)$, and where $a \circ b=a b+b a$.

Kosier had studied rings satisfying (i), (iii) and (iv) and has shown that the simple ones which contain an idempotent $e \neq 1$ are alternative, hence either CayleyDickson vector-matrix algebras or associative rings [1]. In the same paper he also gives an example of a simple, 3-dimensional algebra with identity element satisfying these identities and which is not flexible and therefore not alternative.

Nonassociative division rings may be used to coordinate projective planes and it is therefore of interest to determine the division rings satisfying the above identities. Toward this end we consider initially rings of characteristic different from two, that is rings in which $2 x=0$ implies $x=0$ and satisfying identities (i)-(iv). To this we add

Condition I. Let $R$ be a ring with elements $x, y, z$ such that $(x, y, z)^{2}=0$. Whenever $x, y, z$ lie in a subring $S$ of $R$ which can be generated by two elements then it follows that $(x, y, z)=0$.

Received by the editors June 19, 1970.

AMS 1970 subject classifications. Primary 17D05, 17E05.

Key words and phrases. Alternative ring, nonassociative ring, identities, division ring, Artin's theorem, projective plane.

(1) This work was supported in part by a grant from the U.S. Army Research Office (Durham) and the National Science Foundation (Grant GP 23403).

Copyright (C) 1971, American Mathematical Society 
It is clear, because of Artin's theorem for alternative rings, that Condition I is satisfied in every alternative ring. In this paper we show that the condition is also sufficient to assure that a ring of characteristic not two satisfying identities (i)-(iv) must be alternative. Consequently all such division rings must be alternative and hence either Cayley-Dickson division algebras or associative. In the final part of the paper we show that any ring satisfying (iv) and any two of the first three identities and Condition I must be alternative, so that our results complement the results of Kosier.

Main section. For the remainder of the paper $R$ will denote a ring of characteristic different from two satisfying identities (i)-(iv) and Condition I. In an arbitrary ring the Teichmüller identity $f(w, x, y, z)=(w x, y, z)-(w, x y, z)+(w, x, y z)$ $-w(x, y, z)-(w, x, y) z=0$ is satisfied. Whenever we wish to apply this identity to the elements $a, b, c, d$ in $R$ we shall write $0=f(a, b, c, d)$.

From $0=f(x, x, x, y)$, we obtain $\left(x^{2}, x, y\right)-\left(x, x^{2}, y\right)+(x, x, x y)=x(x, x, y)$ $+(x, x, x) y$. Then $\left(x^{2}, x, y\right)=x \circ(x, x, y)$ follows from (i) while $\left(x, x^{2}, y\right)=$ $x \circ(x, x, y)$ follows from (ii). Hence the first two terms of the left-hand side cancel. Also $(x, x, x) y=0$, as a consequence of (iv). This leaves $(x, x, x y)=x(x, x, y)$. By linearizing (iii), that is replacing $z$ by $z+w$ and cancelling, we see that $(x, y, z \circ w)$ $=w \circ(x, y, z)+z \circ(x, y, w)$. If we put $w=x, y=x$, and $z=y$, in this last identity we see that $(x, x, x \circ y)=x \circ(x, x, y)+y \circ(x, x, x)$. But $(x, x, x)=0$, as a result of (iv), so $(x, x, x \circ y)=x \circ(x, x, y)$. Since we have proved earlier that $(x, x, x y)$ $=x(x, x, y)$, it then follows that $(x, x, y x)=(x, x, y) x$. By going to the antiisomorphic copy of $R$, that is the ring in which we introduce a new multiplication $x * y=y x$, retaining the same addition, we see that $(y x, x, x)=(y, x, x) x$, and $(x y, x, x)=x(y, x, x)$. A linearization of (iv) implies that $(y, x, x)+(x, y, x)$ $+(x, x, y)=0$. Replacing $y$ by $x y$ in this last identity we see that $(x, x y, x)=$ $-(x y, x, x)-(x, x, x y)=-x(y, x, x)-x(x, x, y)=x(x, y, x)$. By going to the antiisomorphic copy of $R$ again we may conclude that $(x, y x, x)=(x, y, x) x$. But then $0=f(x, y, x, x)=(x y, x, x)-(x, y x, x)+\left(x, y, x^{2}\right)-x(y, x, x)-(x, y, x) x=x(y, x, x)$ $-(x, y, x) x+x \circ(x, y, x)-x(y, x, x)-(x, y, x) x$, using (iii) and the identities previously established. Cancelling shows that $x(x, y, x)-(x, y, x) x=0$. Thus

$$
(x,(x, y, x))=0,
$$

where the commutator $(a, b)$ is defined by $(a, b)=a b-b a$. Let $a=(x, y, x)$. Then (1) states that $(x, a)=0$. Replace $y$ by $x \circ y$ in (1). A linearization of (ii) shows that $(x, x \circ y, x)=x \circ(x, y, x)+y \circ(x, x, x)=x \circ(x, y, x)=x \circ a$. Thus $(x, x \circ a)=0$. But $(x, a)=0$ implies that $x \circ a=2 x a$. Thus $2(x, x a)=0$. Since we have characteristic different from two it follows that $(x, x a)=0$. Thus $0=x(x a)-(x a) x=x(a x)-(x a) x$ $=-(x, a, x)$, since $(x, a)=0$. Thus

$$
(x,(x, y, x), x)=0 .
$$


In (2) replace $y$ by $y^{2}$. Since $\left(x, y^{2}, x\right)=y \circ a$, using (ii), we see that $(x, y \circ a, x)=0$. But a linearization of (ii) implies $(x, y \circ a, x)=y \circ(x, a, x)+a \circ(x, y, x)$. Because of (2), $(x, a, x)=0$. Combining the last three identities we see that $0=a \circ a=2 a^{2}$. By hypothesis it follows first that $a^{2}=0$. But then Condition I allows us to conclude that $a=0$, or $(x, y, x)=0$. In other words we have proved

\section{LEMMA $1 . R$ must be flexible.}

A linearization of the flexible law leads to the identity $(x, y, z)=-(z, y, x)$. In subsequent calculations we shall assume the reader is familiar with this calculation as well as with $(x, x, x y)=x(x, x, y),(x, x, y x)=(x, x, y) x,(x y, x, x)=x(y, x, x)$, and $(y x, x, x)=(y, x, x) x$, without a specific reference. Then $0=f(x, x, y, y)$ $=\left(x^{2}, y, y\right)-(x, x y, y)+\left(x, x, y^{2}\right)-x(x, y, y)-(x, x, y) y=x \circ(x, y, y)-(x, x y, y)$ $+y \circ(x, x, y)-x(x, y, y)-(x, x, y) y=-(x, x y, y)+(x, y, y) x+y(x, x, y)$, using (i) and (iii). Thus

$$
(x, x y, y)=(x, y, y) x+y(x, x, y)
$$

Interchanging $x$ and $y$ in (3) and using the linearization of the flexible law it follows that

$$
(x, y x, y)=x(x, y, y)+(x, x, y) y .
$$

Then $0=f(x, y, y, x)=(x y, y, x)-\left(x, y^{2}, x\right)+(x, y, y x)-x(y, y, x)-(x, y, y) x$. As a result of Lemma $1,\left(x, y^{2}, x\right)=0$, and $-x(y, y, x)-(x, y, y) x=x(x, y, y)$ $-(x, y, y) x=-((x, y, y), x)$. Also $(x y, y, x)+(x, y, y x)=(x y, y, x)-(y x, y, x)$ $=((x, y), y, x)$. Thus we have shown

$$
((x, y), y, x)=((x, y, y), x)=-((y, y, x), x) .
$$

A linearization of (i) implies that $(x \circ y, y, x)=x \circ(y, y, x)+y \circ(x, y, x)$. But $y \circ(x, y, x)=0$, as a result of Lemma 1 so that $(x \circ y, y, x)=x \circ(y, y, x)$. Comparing this last identity with (5) and using characteristic different from two we see that

$$
(x y, y, x)=x(y, y, x) \text { and }(y x, y, x)=(y, y, x) x .
$$

Interchanging $x$ and $y$ in (6) it follows that

$$
(y x, x, y)=y(x, x, y) \text { and }(x y, x, y)=(x, x, y) y .
$$

Using Lemma 1 on (7) it follows that $(y, x, y x)=y(y, x, x)$. We shall concern ourselves now with the linearization of this last identity, namely

$$
(y, x, z x)+(z, x, y x)=z(y, x, x)+y(z, x, x) .
$$

In (8) let $z=y x$. Then $(y, x, y x \cdot x)+(y x, x, y x)=y x \cdot(y, x, x)+y(y x, x, x)$. However the second term of the left-hand side vanishes because of Lemma 1. If we let $u=(y, x, x)$, then the right-hand side of the equation equals $y x \cdot u+y \cdot u x$. Thus

$$
(y, x, y x \cdot x)=y x \cdot u+y \cdot u x .
$$


A second linearization of the identity $(y, x, y x)=y(y, x, x)$, which was established just after (7), leads to

$$
(y, x, y z)+(y, z, y x)=y(y, x, z)+y(y, z, x) .
$$

Substitute $z=x^{2}$ in (10). Then $\left(y, x, y \cdot x^{2}\right)+\left(y, x^{2}, y x\right)=y\left(y, x, x^{2}\right)+y\left(y, x^{2}, x\right)$. Using (ii) we may replace $\left(y, x^{2}, y x\right)$ by $x \circ(y, x, y x)$, and $y\left(y, x^{2}, x\right)$ by $y \cdot x(y, x, x)$ $+y \cdot(y, x, x) x$. Using (iii) we may replace $y\left(y, x, x^{2}\right)$ by $y \cdot x(y, x, x)+y \cdot(y, x, x) x$. Hence $\left(y, x, y \cdot x^{2}\right)+x \circ(y, x, y x)=2 y \cdot x u+2 y \cdot u x$. But following (7) we saw that $(y, x, y x)=y(y, x, x)=y u$. Thus $\left(y, x, y \cdot x^{2}\right)+x \circ y u=2 y \cdot x u+2 y \cdot u x$, or

$$
\left(y, x, y \cdot x^{2}\right)=-x \cdot y u-y u \cdot x+2 y \cdot x u+2 y \cdot u x .
$$

Subtracting (11) from (9) we obtain $(y, x, u)=(y, x, y x \cdot x)-\left(y, x, y \cdot x^{2}\right)=y x \cdot u$ $+y \cdot u x+x \cdot y u+y u \cdot x-2 y \cdot x u-2 y \cdot u x=y x \cdot u-y \cdot u x+x \cdot y u+y u \cdot x-2 y \cdot x u$ $=(y, x, u)+(y, u, x)+x \cdot y u-y \cdot x u$. Cancelling one term and using Lemma 1 , this identity becomes

$$
(x, u, y)=x \cdot y u-y \cdot x u .
$$

Let us denote $(x, y)$ by $v=(x, y)$. Then the right-hand side of (12) may be rewritten as $x \cdot y u-y \cdot x u=-(x, y, u)+x y \cdot u+(y, x, u)-y x \cdot u=v u-(x, y, u)+(y, x, u)$. Comparing this with (12) and using Lemma 1 it follows that

$$
v u=(x, y, u)+(u, x, y)+(x, u, y) .
$$

The following is a technical result which we shall apply presently.

LEMMA 2. If $x \circ(y \circ a)=y \circ(x \circ a)$ then $((x, y), a)=2(x, y, a)+2(a, x, y)$ $+2(x, a, y)$.

Proof. By hypothesis $x \cdot y a+x \cdot a y+y a \cdot x+a y \cdot x=y \cdot x a+y \cdot a x+x a \cdot y+a x \cdot y$. This leads to $x \cdot y a+a y \cdot x+(y, a, x)=y \cdot x a+a x \cdot y+(x, a, y)$. But the left-hand side of this last identity equals $-(x, y, a)+x y \cdot a+(a, y, x)+a \cdot y x+(y, a, x)$, while the right-hand side equals $-(y, x, a)+y x \cdot a+(a, x, y)+a \cdot x y+(x, a, y)$. Comparing these and using Lemma 1 we find that $((x, y), a)=2(x, y, a)+2(a, x, y)+2(x, a, y)$. This completes the proof of the lemma.

If we start with $\left(x^{2}, x, y^{2}\right)$ and apply (i) and (iii) to it, but in different orders we see that on the one hand $\left(x^{2}, x, y^{2}\right)=x \circ\left(x, x, y^{2}\right)=x \circ(y \circ u)$, while on the other hand $\left(x^{2}, x, y^{2}\right)=y \circ\left(x^{2}, x, y\right)=y \circ(x \circ u)$. Thus $u$ satisfies the hypothesis of Lemma 2 and thus its conclusion. Hence

$$
(v, u)=2(x, y, u)+2(u, x, y)+2(x, u, y) .
$$

Comparison of (13) with (14) implies

$$
u v=-(x, y, u)-(u, x, y)-(x, u, y)=-v u .
$$

Similarly we may expand $\left(x^{2}, x^{2}, y^{2}\right)=x \circ\left(y \circ\left(x, x^{2}, y\right)\right)=y \circ\left(x \circ\left(x, x^{2}, y\right)\right)$, so 
that $\left(x, x^{2}, y\right)$ satisfies the hypothesis of Lemma 2. But because of (ii), $\left(x, x^{2}, y\right)$ $=x \circ u$. Consequently $x \circ u$ satisfies the conclusion of Lemma 2 and

$$
(v, x \circ u)=2(x, y, x \circ u)+2(x \circ u, x, y)+2(x, x \circ u, y) .
$$

In an arbitrary ring it may be verified that $(a, b \circ c)-b \circ(a, c)-c \circ(a, b)=(b, a, c)$ $+(c, a, b)-(a, b, c)-(c, b, a)-(b, c, a)-(a, c, b)$. However in a flexible ring the right-hand side of the last equation vanishes, so that

$$
(a, b \circ c)=b \circ(a, c)+c \circ(a, b) \text {. }
$$

Applying the linearizations of (iii), (i) and (ii) to the right-hand side of (16) we see that $2(x, y, x \circ u)+2(x \circ u, x, y)+2(x, x \circ u, y)=2 x \circ(x, y, u)+2 u \circ(x, y, x)$ $+2 x \circ(u, x, y)+2 u \circ(x, x, y)+2 x \circ(x, u, y)+2 u \circ(x, x, y)$. Using Lemma 1 it follows that $(x, y, x)=0$, and that $2 u \circ(x, x, y)+2 u \circ(x, x, y)=4 u \circ u=8 u^{2}$. Thus the right-hand side of (16) equals $8 u^{2}+2 x \circ(x, y, u)+2 x \circ(u, x, y)+2 x \circ(x, u, y)$. The left-hand side of (16) equals $x \circ(v, u)+u \circ(v, x)$, as a consequence of (17). Thus $x \circ(v, u)+u \circ(v, x)=8 u^{2}+2 x \circ(x, y, u)+2 x \circ(u, x, y)+2 x \circ(x, u, y)$. On the other hand it follows from (14) that $x \circ(v, u)=2 x \circ(x, y, u)+2 x \circ(u, x, y)$ $+2 x \circ(x, u, y)$. Comparing the last two equations it becomes evident that

$$
8 u^{2}=u \circ(v, x) \text {. }
$$

In (15) we showed that $u \circ v=0$. Thus using (17) we have $0=(u \circ v, x)=u \circ(v, x)$ $+v \circ(u, x)$. This proves that $u \circ(v, x)=-v \circ(u, x)$. However $-(u, x)=-((x, x, y), x)$ $=x(x, x, y)-(x, x, y) x=(x, x, x y)-(x, x, y x)=(x, x, v)$, so that $-v \circ(u, x)$ $=v \circ(x, x, v)=\left(x, x, v^{2}\right)$, using (iii). Summarizing,

$$
8 u^{2}=u \circ(v, x)=-v \circ(u, x)=\left(x, x, v^{2}\right) .
$$

We may combine the two identities of (7) with the linearization of the flexible law to obtain $(y, x,(x, y))=((y, x, x), y)$. One linearization of this identity yields $(y, x,(x, z))+(z, x,(x, y))=((z, x, x), y)+((y, x, x), z)$. In this last equation let $y=v$, and $z=v^{2}$. Then $\left(v, x,\left(x, v^{2}\right)\right)+\left(v^{2}, x,(x, v)\right)=\left(\left(v^{2}, x, x\right), v\right)+\left((v, x, x), v^{2}\right)$. We shall show that the right-hand side of the last equation is zero while the lefthand side equals $2\left(v^{2}, x,(x, v)\right)$. First we note that by putting $b=c=u$, and $a=v$, in (17) that $2\left(v, u^{2}\right)=2 u \circ(v, u)$. Using characteristic different from two it follows that $\left(u^{2}, v\right)=u \circ(u, v)$. On the other hand if we set $a=b=u$, and $c=v$, in (17) then $(u, u \circ v)=u \circ(u, v)+v \circ(u, u)$. Thus $(u, u \circ v)=u \circ(u, v)$. But $u \circ v=0$, as a result of $(15)$ so that $\left(u^{2}, v\right)=u \circ(u, v)=0$. As a consequence of (19) and the linearization of the flexible law we note that $\left(v^{2}, x, x\right)=-8 u^{2}$, implying that $\left(\left(v^{2}, x, x\right), v\right)$ $=-8\left(u^{2}, v\right)=0$. Also setting $a=(v, x, x), b=c=v$ in (17) we see that $2\left((v, x, x), v^{2}\right)$ $=2 v \circ((v, x, x), v)$, implying that $\left((v, x, x), v^{2}\right)=v \circ((v, x, x), v)$. On the other hand if we let $a=b=v$, and $c=(v, x, x)$, in (17) then $(v, v \circ(v, x, x))=v \circ(v,(v, x, x))$ $+(v, x, x) \circ(v, v)$. Therefore $v \circ((v, x, x), v)=(v \circ(v, x, x), v)$. But $v \circ(v, x, x)$ $=\left(v^{2}, x, x\right)$, as a result of (i), while $\left(v^{2}, x, x\right)=-8 u^{2}$, as a result of (19) and the 
linearization of the flexible law. Hence $\left((v, x, x), v^{2}\right)=v \circ((v, x, x), v)=(v \circ(v, x, x), v)$ $=-8\left(u^{2}, v\right)=0$. It is now established that the right-hand side of the equation we are considering is zero. Next setting $a=x, b=c=v$, in (17) and dividing by 2 shows that $\left(x, v^{2}\right)=v \circ(x, v)$. Thus $\left(v, x,\left(x, v^{2}\right)\right)=(v, x, v \circ(x, v))$. By a linearization of (iii), $(v, x, v \circ(x, v))=v \circ(v, x,(x, v))+(x, v) \circ(v, x, v)=v \circ(v, x,(x, v))$, using Lemma 1. But as a result of (i), $v \circ(v, x,(x, v))=\left(v^{2}, x,(x, v)\right)$. Thus the left-hand side of the equation under consideration equals $2\left(v^{2}, x,(x, v)\right)$. We have shown therefore that $2\left(v^{2}, x,(x, v)\right)=0$, so that

$$
\left(v^{2}, x,(x, v)\right)=0 .
$$

The equation immediately following (19) reads $(y, x,(x, y))=((y, x, x), y)$. This time we linearize it twice to obtain

$$
\begin{aligned}
(y, w,(x, z))+( & (y, x,(w, z))+(z, w,(x, y))+(z, x,(w, y)) \\
& =((y, x, w), z)+((y, w, x), z)+((z, x, w), y)+((z, w, x), y) .
\end{aligned}
$$

In (21) we put $y=v^{2}, z=v$, and $w=x^{2}$, to obtain

$$
\begin{aligned}
\left(v^{2}, x^{2},(x, v)\right) & +\left(v^{2}, x,\left(x^{2}, v\right)\right)+\left(v, x^{2},\left(x, v^{2}\right)\right)+\left(v, x,\left(x^{2}, v^{2}\right)\right) \\
= & \left(\left(v^{2}, x, x^{2}\right), v\right)+\left(\left(v^{2}, x^{2}, x\right), v\right)+\left(\left(v, x, x^{2}\right), v^{2}\right)+\left(\left(v, x^{2}, x\right), v^{2}\right) .
\end{aligned}
$$

As a result of (17) it follows first that $\left(a, v^{2}\right)=v \circ(a, v)$, and then that $v \circ(a, v)$ $=(v \circ a, v)$. Using (ii) and (iii) it is clear that $\left(v, x, x^{2}\right)=x \circ(v, x, x)=\left(v, x^{2}, x\right)$ and $\left(v^{2}, x, x^{2}\right)=x \circ\left(v^{2}, x, x\right)=\left(v^{2}, x^{2}, x\right)$. Thus $\left(\left(v, x^{2}, x\right), v^{2}\right)=\left(\left(v, x, x^{2}\right), v^{2}\right)=$ $\left(v \circ\left(v, x, x^{2}\right), v\right)$. But $v \circ\left(v, x, x^{2}\right)=\left(v^{2}, x, x^{2}\right)$, using (i). It is now clear, making similar calculations, that the right-hand side of (22) equals $4\left(\left(v^{2}, x, x^{2}\right), v\right)$. Using (ii) and (20) it is clear that $\left(v^{2}, x^{2},(x, v)\right)=x \circ\left(v^{2}, x,(x, v)\right)=0$. Also because of (17) $\left(\dot{x}, v^{2}\right)=v \circ(x, v)$. Thus $\left(v, x,\left(x, v^{2}\right)\right)=(v, x, v \circ(x, v))=v \circ(v, x,(x, v))+(x, v) \circ(v, x, v)$, using a linearization of (iii). But $(v, x, v)=0$, using Lemma 1 , while $v \circ(v, x,(x, v))$ $=\left(v^{2}, x,(x, v)\right)=0$, using (i) and (20), so $\left(v, x,\left(x, v^{2}\right)\right)=0$. Hence using (ii), $\left(v, x^{2},\left(x, v^{2}\right)\right)=x \circ\left(v, x,\left(x, v^{2}\right)\right)=0$. This eliminates the first and third terms of the left-hand side of (22). Again $\left(x^{2}, v^{2}\right)=v \circ\left(x^{2}, v\right)$, as a result of (17). But then $\left(v, x,\left(x^{2}, v^{2}\right)\right)=\left(v, x, v \circ\left(x^{2}, v\right)\right)=v \circ\left(v, x,\left(x^{2}, v\right)\right)+\left(x^{2}, v\right) \circ(v, x, v)$, using a linearization of (iii). Because of Lemma $1(v, x, v)=0$. Thus $\left(v, x,\left(x^{2}, v^{2}\right)\right)=v \circ\left(v, x,\left(x^{2}, v\right)\right)$. However (i) implies that $v \circ\left(v, x,\left(x^{2}, v\right)\right)=\left(v^{2}, x,\left(x^{2}, v\right)\right)$. Thus the left-hand side of (22) becomes equal to $2\left(v^{2}, x,\left(x^{2}, v\right)\right)$. Since the right-hand side was seen to equal $4\left(\left(v^{2}, x, x^{2}\right), v\right)$, we may divide by 2 and obtain

$$
\left(v^{2}, x,\left(x^{2}, v\right)\right)=2\left(\left(v^{2}, x, x^{2}\right), v\right) .
$$

Because of $(17),\left(x^{2}, v\right)=x \circ(x, v)$. Thus the left-hand side of (23) equals $\left(v^{2}, x, x \circ(x, v)\right)$. As a result of linearizing (iii), $\left(v^{2}, x, x \circ(x, v)\right)=x \circ\left(v^{2}, x,(x, v)\right)$ $+(x, v) \circ\left(v^{2}, x, x\right)$. But then using (20) and (19) it is clear that the left-hand side of (23) equals $-8(x, v) \circ u^{2}$. Also using $(17),\left(u^{2}, v\right)=u \circ(u, v)=(u, u \circ v)$. But $u \circ v=0$, 
as a result of $(15)$ so $\left(u^{2}, v\right)=0$. But then $\left(\left(v^{2}, x, x^{2}\right), v\right)=\left(x \circ\left(v^{2}, x, x\right), v\right)$, using (iii). Since $\left(v^{2}, x, x\right)=-8 u^{2}$, using (19), we have $\left(x \circ\left(v^{2}, x, x\right), v\right)=-8\left(x \circ u^{2}, v\right)$. But $\left(x \circ u^{2}, v\right)=x \circ\left(u^{2}, v\right)+u^{2} \circ(x, v)$, using (17), so that $\left(x \circ u^{2}, v\right)=u^{2} \circ(x, v)$. Thus $\left(\left(v^{2}, x, x^{2}\right), v\right)=-8 u^{2} \circ(x, v)$, and so the right-hand side of (23) equals $-16 u^{2} \circ(x, v)$. Comparing the two sides of (23) and dividing by 2 , it follows that

$$
u^{2} \circ(x, v)=0 .
$$

Now let $t=(x, v)$. Then part of (19) may be rewritten as

$$
-8 u^{2}=u \circ t .
$$

Then multiplying (25) through by $u$ on the left one obtains $-8 u^{3}=u(u t+t u)=u(u t)$ $+u(t u)=u(u t)+(u t) u$, using Lemma 1. Multiplying (25) through on the right by $u$ we obtain $-8 u^{3}=(u t+t u) u=(u t) u+(t u) u$. Comparison of the last two equations shows that $u(u t)=(t u) u$. Using this and a linearization of the flexible law we see that $0=(u, u, t)+(t, u, u)=\left(u^{2}\right) t-u(u t)+(t u) u-t\left(u^{2}\right)=\left(u^{2}\right) t-t\left(u^{2}\right)$. On the other hand (24) shows us that $\left(u^{2}\right) t+t\left(u^{2}\right)=0$. Comparing the last two equations and using characteristic different from two leads to

$$
u^{2} t=0=t u^{2} .
$$

Using (i) and (ii) it follows that $\left(u^{2}, u, t\right)=u \circ(u, u, t)=\left(u, u^{2}, t\right)$. But $\left(u^{2}, u, t\right)$ $=u^{3} t-u^{2}(u t)$, while $\left(u, u^{2}, t\right)=u^{3} t-u\left(u^{2} t\right)=u^{3} t$, using (26). Comparing the last two equations it is clear that

$$
u^{2}(u t)=0 \text {. }
$$

Using (i) and Lemma $1,\left(u^{2}, t, u\right)=u \circ(u, t, u)=0$. But $\left(u^{2}, t, u\right)=\left(u^{2} t\right) u-u^{2}(t u)$ $=-u^{2}(t u)$, using (26). Thus

$$
u^{2}(t u)=0 \text {. }
$$

Using (25), (27) and (28) it follows that $-8 u^{4}=u^{2}\left(-8 u^{2}\right)=u^{2}(u \circ t)=u^{2}(u t)+u^{2}(t u)$ $=0$. Cancelling by 2 , we obtain

$$
u^{4}=0 \text {. }
$$

Let $r=8 u^{2}$. Then clearly (29) implies that $r^{2}=0$. On the other hand, because of (19) $r$ is also equal to $\left(x, x, v^{2}\right)$. Therefore Condition $\mathrm{I}$ applies and so $r=0$. But this means $8 u^{2}=0$. Since the characteristic is different from two we deduce that $u^{2}=0$. Again Condition $\mathrm{I}$ applies to $u$ and so $u=0$. Since $u=(x, x, y)$, we have shown that $R$ is left alternative. $R$ is already known to be flexible and therefore must be alternative. We have proved

THEOREM 1. In a ring of characteristic not two, satisfying identities (i)-(iv), Condition I is necessary and sufficient for the ring to be alternative.

Next we shall concentrate our attention on a ring $S$ which satisfies identities (ii)-(iv) and Condition I and has characteristic different from two. Then $f(y, x, x, x)$ 
$=(y x, x, x)-\left(y, x^{2}, x\right)+\left(y, x, x^{2}\right)-y(x, x, x)-(y, x, x) x=(y x, x, x)-x \circ(y, x, x)$ $+x \circ(y, x, x)-(y, x, x) x=(y x, x, x)-(y, x, x) x$, using (ii), (iii) and (iv). Hence

$$
(y x, x, x)=(y, x, x) x \text {. }
$$

If we linearize (iv) once we see that $(z, x, x)+(x, z, x)+(x, x, z)=0$. Let $z=x \circ y$. Then $(x \circ y, x, x)=-(x, x \circ y, x)-(x, x, x \circ y)=-x \circ(x, y, x)-y \circ(x, x, x)$ $-x \circ(x, x, y)-y \circ(x, x, x)$, using linearizations of (ii) and (iii). But then because of (iv) we obtain $(x \circ y, x, x)=-x \circ(x, x, y)-x \circ(x, y, x)=x \circ(y, x, x)$, again using a linearization of (iv). Comparing this last identity with (30) it follows that

$$
(x y, x, x)=x(y, x, x) \text {. }
$$

Then $0=f(x, y, x, x)=(x y, x, x)-(x, y x, x)+\left(x, y, x^{2}\right)-x(y, x, x)-(x, y, x) x$. Using (31) we have $(x y, x, x)=x(y, x, x)$, and as a result of (iii), $\left(x, y, x^{2}\right)$ $=x \circ(x, y, x)$. Substituting we obtain, after cancellation,

$$
(x, y x, x)=x(x, y, x) \text {. }
$$

By a linearization of (ii) we have $(x, x \circ y, x)=x \circ(x, y, x)+y \circ(x, x, x)$ $=x \circ(x, y, x)$, using (iv). Comparing this last identity with (32) it follows that

$$
(x, x y, x)=(x, y, x) x .
$$

Now let $p=(x, y, x)$. Then $(x, p, x)=(x, x y \cdot x-x \cdot y x, x)=x(x, x y, x)-(x, y x, x) x$ $=x(p x)-(x p) x=-(x, p, x)$, using (32) and (33). But this implies $2(x, p, x)=0$, so that

$$
(x, p, x)=0 .
$$

In (34) replace $y$ by $y^{2}$. Then $0=\left(x,\left(x, y^{2}, x\right), x\right)=(x, y \circ p, x)$, using (ii). But using a linearization of (ii), $(x, y \circ p, x)=y \circ(x, p, x)+p \circ(x, y, x)=2 p^{2}$, because of (34). Thus $2 p^{2}=0$. But then using characteristic different from two we obtain $p^{2}=0$, and so Condition I implies that $p=0$. Thus $S$ is flexible. From the linearization of the flexible law and (iii) it is now possible to deduce (i), since $\left(x^{2}, y, z\right)=-\left(z, y, x^{2}\right)$ $=-x \circ(z, y, x)=x \circ(x, y, z)$. But now $S$ satisfies the conditions of Theorem 1 and hence must be alternative. We have proved

THEOREM 2. In a ring of characteristic different from two, satisfying identities (ii)-(iv), Condition I is necessary and sufficient for the ring to be alternative.

For the remainder of the paper we consider a ring $T$ of characteristic different from two, satisfying identities (i), (iii) and (iv), and Condition I. Then $0=f(x, x, y, x)=\left(x^{2}, y, x\right)-(x, x y, x)+(x, x, y x)-x(x, y, x)-(x, x, y) x$. Comparing this with $\left(x^{2}, y, x\right)=x \circ(x, y, x)$, which follows from (i), we see that $(x, y, x) x$ $-(x, x y, x)+(x, x, y x)=(x, x, y) x$. By a linearization of (iv) we see that $-(x, x y, x)$ $=(x y, x, x)+(x, x, x y)$. With this substitution it follows that $(x, y, x) x+(x y, x, x)$ $+(x, x, x y+y x)=(x, x, y) x$. But a linearization of (iii) implies $(x, x, x y+y x)$ 
$=x \circ(x, x, y)$. With this substitution it follows that $(x, y, x) x+x(x, x, y)+(x y, x, x)$ $=0$. Thus

$$
\begin{aligned}
(x y, x, x) & =-x(x, x, y)-(x, y, x) x=(x,(x, y, x))-x(x, y, x)-x(x, x, y) \\
& =(x,(x, y, x))+x(y, x, x) .
\end{aligned}
$$

From a linearization of (i) it follows that $(x \circ y, x, x)=x \circ(y, x, x)+y,(x, x, x)$ $=x \circ(y, x, x)$, because of (iv). Comparing the last identity with (35) implies

$$
(y x, x, x)=-(x,(x, y, x))+(y, x, x) x=-x(x, y, x)-(x, x, y) x .
$$

The anti-isomorphic copy of $T$ satisfies the same identities so that the next two identities follow from (35) and (36).

$$
\begin{aligned}
& (x, x, y x)=-(x,(x, y, x))+(x, x, y) x . \\
& (x, x, x y)=(x,(x, y, x))+x(x, x, y) .
\end{aligned}
$$

Since $0=f(x, x, x, y)=\left(x^{2}, x, y\right)-\left(x, x^{2}, y\right)+(x, x, x y)-x(x, x, y)-(x, x, x) y$, and $(x, x, x)=0$ from (iv), and $\left(x^{2}, x, y\right)=x \circ(x, x, y)$ from (i), we obtain after cancellation $x \circ(x, x, y)-\left(x, x^{2}, y\right)+(x, x, x y)-x(x, x, y)=0$. Comparing the last equation with (38) leads to

$$
\left(x, x^{2}, y\right)=x \circ(x, x, y)+(x,(x, y, x)) .
$$

By going to the anti-isomorphic copy of $T$ we get from (39) that

$$
\left(y, x^{2}, x\right)=x \circ(y, x, x)-(x,(x, y, x)) .
$$

Define $q=(x, y, x)$. Then $(x, x, q)=(x, x, x y \cdot x-x \cdot y x)=-(x,(x, x y, x))+(x, x, x y) x$ $-(x,(x, y x, x))-x(x, x, y x)$, using (37) with $x y$ in place of $y$ and using (38) with $y x$ in place of $y$. Now $-(x,(x, x y, x))-(x,(x, y x, x))=-(x,(x, x \circ y, x))$. But $(x, x \circ y, x)=-(x \circ y, x, x)-(x, x, x \circ y)=-x \circ(y, x, x)-x \circ(x, x, y)=x \circ(x, y, x)$, using linearizations of (i), (iii) and (iv). Thus $-(x,(x, x y, x))-(x,(x, y x, x))$ $=-(x, x \circ q)$. Then $(x, x, x y) x=(x, q) x+x(x, x, y) \cdot x$, using $(38)$, while $-x(x, x, y x)$ $=x(x, q)-x \cdot(x, x, y) x$, as a consequence of (37). Collecting terms we find that $(x, x, q)=-(x, x \circ q)+(x, q) x+x(x, x, y) \cdot x+x(x, q)-x \cdot(x, x, y) x=(x,(x, x, y), x)$ $-(x, x q+q x)+x(x, q)+(x, q) x$. But expanding $-(x, x q+q x)+x(x, q)+(x, q) x$ $=-x(x q)-x(q x)+(x q) x+(q x) x+x(x q)-x(q x)+(x q) x-(q x) x=2(x, q, x)$. Combining the last two equations we see that

$$
(x, x, q)=(x,(x, x, y), x)+2(x, q, x) .
$$

Going to the anti-isomorphic copy of $T$, (41) becomes

$$
(q, x, x)=(x,(y, x, x), x)+2(x, q, x) .
$$

Adding equations (41) and (42) and using the linearization of (iv) we see that

$$
\begin{aligned}
-(x, q, x) & =(x, x, q)+(q, x, x)=(x,(x, x, y)+(y, x, x), x)+4(x, q, x) \\
& =-(x,(x, y, x), x)+4(x, q, x)=3(x, q, x) .
\end{aligned}
$$


But then $4(x, q, x)=0$, so that use of characteristic different from two implies

$$
(x, q, x)=0 .
$$

Replacing $y$ by $y^{2}$ in (43),

$$
\begin{aligned}
0 & =\left(x,\left(x, y^{2}, x\right), x\right)=-\left(x,\left(y^{2}, x, x\right)+\left(x, x, y^{2}\right), x\right) \\
& =-(x, y \circ(y, x, x)+y \circ(x, x, y), x) \\
& =(x, y \circ(x, y, x), x)=(x, y \circ q, x),
\end{aligned}
$$

using the linearizations of (i), (iii) and (iv). But for the same reasons $(x, y \circ q, x)$ $=-(y \circ q, x, x)-(x, x, y \circ q)=-y \circ(q, x, x)-q \circ(y, x, x)-y \circ(x, x, q)-q \circ(x, x, y)$ $=y \circ(x, q, x)+q \circ(x, y, x)$. But (43) makes the first term vanish, while $(x, y, x)=q$, so that $0=2 q^{2}$. Using characteristic different from two it follows that $q^{2}=0$, so that $q=0$, because of Condition I. We have shown

$$
(x, y, x)=0 \text {, }
$$

or the flexible law. Now $0=f(x, y, y, x)=(x y, y, x)-\left(x, y^{2}, x\right)+(x, y, y x)-x(y, y, x)$ $-(x, y, y) x$. But $-\left(x, y^{2}, x\right)=0$, because of $(44)$, while $(x y, y, x)+(x, y, y x)$ $=(x y, y, x)-(y x, y, x)$, as a result of the linearization of (44). Thus $(x y-y x, y, x)$ $=x(y, y, x)-(y, y, x) x$. On the other hand $(x y+y x, y, x)=x \circ(y, y, x)+y \circ(x, y, x)$ $=x \circ(y, y, x)$, using (44) and the linearization of (i). If we compare the last two equations we find that $2(x y, y, x)=2 x(y, y, x)$. Using characteristic different from two it follows that

$$
(x y, y, x)=x(y, y, x) .
$$

We linearize (45), replacing $x$ by $x+z$ and by $x-z$ and then comparing the results. This leads to

$$
(x y, y, z)+(z y, y, x)=z(y, y, x)+x(y, y, z) .
$$

Also $0=f(x, y, y, z)=(x y, y, z)-\left(x, y^{2}, z\right)+(x, y, y z)-x(y, y, z)-(x, y, y) z$. In this equation replace $(x y, y, z)$ by solving for it in (46) and using the linearization of (44). Thus $0=-(y \circ z, y, x)-\left(x, y^{2}, z\right)+z \circ(y, y, x)$. But $-(y \circ z, y, x)=-y \circ(z, y, x)$ $-z \circ(y, y, x)$, using a linearization of (i). Solving the last two equations simultaneously we arrive at $\left(x, y^{2}, z\right)=-y \circ(z, y, x)=y \circ(x, y, z)$, using the linearization of (44). Thus

$$
\left(x, y^{2}, z\right)=y \circ(x, y, z)
$$

Note that (47) is the same identity as (ii). Hence we can use Theorem 1 to obtain the result that $T$ is alternative. We have proved

THEOREM 3. In a ring of characteristic different from two, satisfying identities (i), (iii) and (iv), Condition I is necessary and sufficient for the ring to be alternative. 
If one wishes to obtain the same results for rings satisfying (i), (ii) and (iv) one need only consider the anti-isomorphic copy of the ring, which satisfies (ii), (iii) and (iv), in order to obtain the desired conclusion.

The applications of these results are to rings which satisfy the various combinations of identities as well as Condition I. It turns out that a ring without nilpotent elements other than zero satisfies Condition I, as does a division ring. Hence we have shown

MAIN THEOREM. If a ring of characteristic different from two satisfies (iv) and any two of the identities (i)-(iii), then Condition I is necessary and sufficient for the ring to be alternative.

\section{REFERENCE}

1. Frank Kosier, A generalization of alternative rings, Trans. Amer. Math. Soc. 112 (1964), 32-42. MR 29 \#3515.

\section{UNIVERSITY OF IOWA,}

IOWA CITY, IOWA 52240 